\title{
THE NEGATIVE RELATIVISTIC TODA HIERARCHY AND RATIONAL POISSON BRACKETS
}

\author{
J. M. NUNES DA COSTA AND P. A. DAMIANOU
}

\begin{abstract}
We define hierarchies for negative values of the index of Poisson tensors, master symmetries and Hamiltonians for the finite, nonperiodic, relativistic Toda lattice. We show that the usual relations between master symmetries, Poisson tensors and invariants hold over all integer values of the index.
\end{abstract}

KeYwords: Relativistic Toda lattice, bi-Hamiltonian system, Poisson brackets, master symmetries.

AMS SubJeCt Classification (2000): 37J15, 37J35 and 70H06.

\section{Introduction}

The relativistic Toda lattice was introduced by S. N. Ruijsenaars [13] and was investigated in [1], [11], [12], [2] and [9]. In terms of canonical coordinates the relativistic Toda lattice is defined by the Hamiltonian

$$
H\left(q^{1}, \ldots, q^{N}, p_{1}, \ldots, p_{N}\right)=\sum_{j=1}^{N} e^{p_{j}} f\left(q^{j-1}-q^{j}\right) f\left(q^{j}-q^{j+1}\right)
$$

where $f(x)=\sqrt{1+g^{2} e^{x}}$ and, by convention, $q^{0}=-\infty, q^{N+1}=\infty$. The number $g$ is a coupling constant. To see the connection with the nonrelativistic Toda lattice one writes the equation in Newtonian form

$$
\ddot{q}^{j}=g^{2} \dot{q}^{j}\left(\dot{q}^{j-1} \frac{e^{\left(q^{j-1}-q^{j}\right)}}{1+g^{2} e^{\left(q^{j-1}-q^{j}\right)}}-\dot{q}^{j+1} \frac{e^{\left(q^{j}-q^{j+1}\right)}}{1+g^{2} e^{\left(q^{j}-q^{j+1}\right)}}\right),
$$

$j=1,2, \ldots, N$. Setting $\dot{q}^{j}=\dot{Q}_{j}+c$ and letting $c \rightarrow \infty$ and $g \rightarrow 0$ in such a way that $g c=1$, one obtains the equations of motion for the classical Toda lattice

$$
\ddot{Q}_{j}=e^{Q_{j-1}-Q_{j}}-e^{Q_{j}-Q_{j+1}} .
$$

\footnotetext{
J. M. Nunes da Costa supported by Centro de Matemática da Universidade de Coimbra.
} 
In the classical case one uses a change of variables to prove integrability. We follow the same technique. Combining the changes of variables from [13], [1], we set

$$
\begin{aligned}
& a_{j}=g^{2} e^{q^{j}-q^{j+1}+p_{j}} f\left(q^{j-1}-q^{j}\right) / f\left(q^{j}-q^{j+1}\right) \\
& b_{j}=\dot{q}^{j}-a_{j}
\end{aligned}
$$

In these variables the Hamiltonian is homogeneous quadratic and the equations of motion become

$$
\left\{\begin{array}{l}
\dot{a}_{i}=a_{i}\left(b_{i+1}-b_{i}+a_{i+1}-a_{i-1}\right) \\
\dot{b_{i}}=b_{i}\left(a_{i}-a_{i-1}\right)
\end{array}\right.
$$

where $i=1, \ldots, N$ and, by convention, $a_{0}=a_{N}=0$.

Following [2] we write these equations in Lax pair form $(L, M)$ where

$$
L=\left(\begin{array}{cccccc}
a_{1}+b_{1} & a_{1} & 0 & \cdots & \cdots & 0 \\
a_{2}+b_{2} & a_{2}+b_{2} & a_{2} & 0 & \cdots & 0 \\
a_{3}+b_{3} & a_{3}+b_{3} & a_{3}+b_{3} & a_{3} & \cdots & 0 \\
\vdots & & \ddots & \ddots & & \vdots \\
a_{N-1}+b_{N-1} & \ldots & & \ddots & a_{N-1}+b_{N-1} & a_{N-1} \\
b_{N} & b_{N} & \cdots & \cdots & & b_{N}
\end{array}\right)
$$

and

$$
M=\left(\begin{array}{cccccc}
0 & a_{1} & 0 & \cdots & \cdots & 0 \\
0 & -a_{1} & a_{2} & \cdots & & \vdots \\
0 & 0 & -a_{2} & a_{3} & \cdots & \\
\vdots & & \ddots & \ddots & \ddots & \vdots \\
\vdots & & & \ddots & \ddots & a_{N-1} \\
0 & \cdots & \cdots & & 0 & -a_{N-1}
\end{array}\right)
$$

This shows that the functions $\bar{H}_{n-1}=\frac{1}{n} \operatorname{Tr} L^{n}$ are constants of motion and therefore the system is integrable.

In the new coordinates $a_{j}, b_{j}$ the symplectic bracket is transformed into a new quadratic Poisson bracket defined as follows: 


$$
\begin{aligned}
\left\{a_{i}, a_{i+1}\right\} & =-a_{i} a_{i+1} \\
\left\{a_{i}, b_{i}\right\} & =a_{i} b_{i} \\
\left\{a_{i}, b_{i+1}\right\} & =-a_{i} b_{i+1} .
\end{aligned}
$$

All other brackets are zero. This bracket has $\operatorname{det} L=\prod_{i=1}^{N} b_{i}$ as Casimir, and the eigenvalues of $L$ are in involution. We denote this bracket by $\pi_{1}$. We next define a linear bracket $\pi_{0}$ as follows:

$$
\begin{aligned}
\left\{a_{i}, b_{i}\right\} & =a_{i} \\
\left\{a_{i}, b_{i+1}\right\} & =-a_{i} \\
\left\{b_{i}, b_{i+1}\right\} & =a_{i} .
\end{aligned}
$$

All other brackets are zero. In this bracket $\operatorname{Tr} L$ is the Casimir and $\bar{H}_{1}=$ $\frac{1}{2} \operatorname{Tr} L^{2}$ is the Hamiltonian. Therefore we have a bi-Hamiltonian system, a situation similar to the classical case. The bi-Hamiltonian formulation and the complete integrability of this system were established using different methods such as the Lax representation [2], master symmetries [11], [2] and recursion operators [1], [11]. Another approach to obtain the multi-Hamiltonian formulation for the relativistic Toda lattice, using Oevel's theorem [10] and based on a method introduced by Das and Okubo [5] and Fernandes [6], was adopted in [9]. In that work a recursion operator is defined in the $(q, p)$ space, the resulting sequence of Poisson tensors, master symmetries and invariants are then projected in the space of $(a, b)$ variables to construct the usual multi-Hamiltonian hierarchies. The initial brackets of the hierarchy are $\pi_{0}$ and $\pi_{1}$ constructed above.

In this paper we extend to negative values of the index the usual hierarchies of Poisson tensors, master symmetries and Hamiltonians, for the finite nonperiodic relativistic Toda lattice. The extension is obtained in natural $(p, q)$ coordinates and also in $(a, b)$ Flaschka coordinates. This procedure was already adopted in [3] for the finite non-periodic Toda lattice and we mimic the techniques of that paper. As was pointed out in [3] the negative hierarchy was proven useful, for example, in establishing the bi-Hamiltonian formulation of the Bogoyavlensky-Toda systems [4].

The paper is divided into 2 parts. In the first part (section 2) we recall the main results on the finite, nonperiodic relativistic Toda lattice, including the well-known positive hierarchies of Poisson tensors, master symmetries 
and Hamiltonians. In section 3 we introduce the negative relativistic Toda hierarchy, in $(p, q)$ and $(a, b)$ coordinates, and show that the relations between master symmetries, Poisson tensors and Hamiltonians, known for the positive hierarchies, hold for all integers values of the index, in both coordinates. We close with some additional results and give, for small dimensions, examples of the rational brackets and master symmetries.

\section{The Relativistic Toda Lattice}

We recall a theorem due to Oevel [10] that gives a method of generating master symmetries for non-degenerate bi-Hamiltonian systems.

Let $\left(M, \Lambda_{0}, \Lambda_{1}\right)$ be a non-degenerate bi-Hamiltonian manifold with a recursion operator $\mathcal{R}=\Lambda_{1} \Lambda_{0}^{-1}$ and let $Y_{1}=\Lambda_{0} \nabla H_{1}=\Lambda_{1} \nabla H_{0}$ be a bi-Hamiltonian vector field on $M$, where $\Lambda_{i}$ denotes also the matrix of the Poisson tensor $\Lambda_{i}$. We denote by $Y_{i}$ the Hamiltonian vector field $\Lambda_{i} \nabla H_{0}$.

Theorem 2.1. Suppose that the vector field $X_{0}$ is a conformal symmetry for both $\Lambda_{0}, \Lambda_{1}$ and $H_{0}$. i.e., for some scalars $\alpha, \beta$, and $\gamma$ we have

$$
\mathcal{L}_{X_{0}} \Lambda_{0}=\alpha \Lambda_{0}, \quad \mathcal{L}_{X_{0}} \Lambda_{1}=\beta \Lambda_{1}, \quad X_{0}\left(H_{0}\right)=\gamma H_{0} .
$$

Then the vector fields $X_{i}=\mathcal{R}^{i} X_{0}$ are master symmetries, the tensors $\Lambda_{i}=$ $\mathcal{R}^{i} \Lambda_{0}$ are Poisson and we have, for $i, j=0,1,2, \ldots$
(a) $\left[X_{i}, X_{j}\right]=(\beta-\alpha)(j-i) X_{i+j}$;
(b) $\left[X_{i}, Y_{j}\right]=(\beta+\gamma+(\beta-\alpha)(j-1)) Y_{i+j}$;
(c) $\mathcal{L}_{X_{i}} \Lambda_{j}=(\beta+(\beta-\alpha)(j-i-1)) \Lambda_{i+j}$;
(d) $X_{i}\left(H_{j}\right)=(\lambda+(\beta-\alpha)(j+i)) H_{i+j}$.

Let us now briefly recall some results on the relativistic Toda lattice following [2], [9], [12]. We consider $\mathbb{R}^{2 N}$ with coordinates $\left(q^{1}, \ldots, q^{N}, p_{1}, \ldots, p_{N}\right)$. Let $\Lambda_{0}$ be the Poisson tensor given by

$$
\begin{aligned}
\Lambda_{0}= & \sum_{i=1}^{N} e^{-p_{i}} \frac{\partial}{\partial q^{i}} \wedge\left(\frac{\partial}{\partial p_{i}}+\sum_{j=i+1}^{N} \frac{\partial}{\partial q^{j}}\right)+\sum_{i=1}^{N-1} e^{q^{i}-q^{i+1}-p_{i+1}}\left(\left(\frac{\partial}{\partial p_{i}}+\frac{\partial}{\partial q^{i+1}}\right)\right. \\
& \left.\wedge\left(\frac{\partial}{\partial p_{i+1}}+\sum_{j=i+2}^{N} \frac{\partial}{\partial q^{j}}\right)-\frac{\partial}{\partial p_{i+1}} \wedge \sum_{j=i+2}^{N} \frac{\partial}{\partial q^{j}}\right)
\end{aligned}
$$


and $\Lambda_{1}$ be the canonical Poisson tensor,

$$
\Lambda_{1}=\sum_{i=1}^{N} \frac{\partial}{\partial q^{i}} \wedge \frac{\partial}{\partial p_{i}}
$$

In [9] it was proved that these Poisson tensors are compatible and also that $X=\Lambda_{0} \nabla H_{1}=\Lambda_{1} \nabla H_{0}$ is a bi-Hamiltonian vector field, with

$$
H_{0}=\sum_{i=1}^{N}\left(e^{q^{i}-q^{i+1}+p_{i}}+e^{p_{i}}\right)
$$

and

$$
H_{1}=\sum_{i=1}^{N}\left(\frac{1}{2}\left(e^{q^{i}-q^{i+1}+p_{i}}+e^{p_{i}}\right)^{2}+e^{q^{i-1}-q^{i}+p_{i-1}}\left(e^{q^{i}-q^{i+1}+p_{i}}+e^{p_{i}}\right)\right) .
$$

By convention, $q^{0}=-\infty$ and $q^{N+1}=+\infty$.

Consider the Flaschka-type transformation $F: \mathbb{R}^{2 N} \rightarrow \mathbb{R}^{2 N-1}[12]$, [9],

$$
F:\left(q^{1}, \ldots, q^{N}, p_{1}, \ldots, p_{N}\right) \mapsto\left(a_{1}, \ldots, a_{N-1}, b_{1}, \ldots, b_{N}\right),
$$

where $a_{i}=e^{q^{i}-q^{i+1}+p_{i}}$ and $b_{i}=e^{p_{i}}$. These are new coordinates in $\mathbb{R}^{2 N-1}$.

The Poisson tensors $\Lambda_{0}$ and $\Lambda_{1}$ project by $F$ onto bivectors on $\mathbb{R}^{2 N-1}$,

$$
\pi_{0}=\sum_{i=1}^{N-1} a_{i}\left(\frac{\partial}{\partial a_{i}} \wedge\left(\frac{\partial}{\partial b_{i}}-\frac{\partial}{\partial b_{i+1}}\right)+\frac{\partial}{\partial b_{i}} \wedge \frac{\partial}{\partial b_{i+1}}\right)
$$

and

$$
\pi_{1}=\sum_{i=1}^{N-1} a_{i} \frac{\partial}{\partial a_{i}} \wedge\left(-a_{i+1} \frac{\partial}{\partial a_{i+1}}+b_{i} \frac{\partial}{\partial b_{i}}-b_{i+1} \frac{\partial}{\partial b_{i+1}}\right) .
$$

These are the Poisson tensors that we defined earlier and they provide a bi-Hamiltonian structure for the relativistic Toda lattice, with Hamiltonians 


$$
\bar{H}_{0}=\sum_{i=1}^{N}\left(a_{i}+b_{i}\right) \quad \text { and } \quad \bar{H}_{1}=\sum_{i=1}^{N}\left(a_{i-1}\left(a_{i}+b_{i}\right)+\frac{1}{2}\left(a_{i}+b_{i}\right)^{2}\right),
$$

where, by convention, $a_{0}=a_{N}=0$. For $i \geq 0, \bar{H}_{i}=\frac{1}{i+1} \operatorname{tr} L^{i+1}$ are constants of motion. The Poisson bracket associated with $\pi_{0}$, which is linear, is the one given by (9), while the Poisson bracket corresponding to $\pi_{1}$ is quadratic and is given by (8).

Coming back to the natural $(p, q)$ coordinates, since $\Lambda_{0}$ (and also $\Lambda_{1}$ ) is non-degenerate, we can define a recursion operator $\mathcal{R}=\Lambda_{1}\left(\Lambda_{0}\right)^{-1}$. Once we have a recursion operator $\mathcal{R}$, an infinite sequence of pairwise compatible Poisson tensors on $\mathbb{R}^{2 N}, \Lambda_{i}=\mathcal{R}^{i} \Lambda_{0}$, and an infinite sequence of Hamiltonians $H_{i}$, given by $d H_{i}={ }^{t} \mathcal{R}\left(d H_{i-1}\right)$ are obtained.

We take

$$
X_{0}=\sum_{i=1}^{N} \frac{\partial}{\partial p_{i}}
$$

and compute

$$
\mathcal{L}\left(X_{0}\right) \Lambda_{0}=-\Lambda_{0}, \quad \mathcal{L}\left(X_{0}\right) \Lambda_{1}=0 \quad \text { and } \quad X_{0}\left(H_{0}\right)=H_{0} .
$$

So Oevel's theorem can be applied with $\alpha=-1, \beta=0$ and $\gamma=1$ [9]. We quote the results for RTL, in natural $(p, q)$ coordinates, in the following theorem.

Theorem 2.2. $\quad$ i) $\Lambda_{i}=\mathcal{R}^{i} \Lambda_{0}$ are Poisson tensors, $i \geq 0$;

ii) the functions $H_{i}, i \geq 0$, are in involution with respect to all $\Lambda_{j}$;

iii) $X_{i}\left(H_{j}\right)=(1+i+j) H_{i+j}, \quad i, j \geq 0$;

iv) $\mathcal{L}_{X_{i}} \Lambda_{j}=(j-i-1) \Lambda_{i+j}, \quad i, j \geq 0$;

v) $\left[X_{i}, X_{j}\right]=(j-i) X_{i+j}, \quad i, j \geq 0$

vi) $\Lambda_{j} \nabla H_{i}=\Lambda_{j-1} \nabla H_{i+1}, \quad i \geq 0, j \geq 1$.

The infinite sequence $\left(\Lambda_{i}\right), i \in \mathbb{N}_{0}$, of higher order Poisson tensors on $\mathbb{R}^{2 N}$ reduce, by $F$, to an infinite sequence $\left(\pi_{i}\right), i \in \mathbb{N}_{0}$, of pairwise compatible Poisson tensors on $\mathbb{R}^{2 N-1}$. Moreover, the master symmetries $X_{i}=\mathcal{R}^{i} X_{0}$, $i \in \mathbb{N}$, are projectable vector fields [9]. We denote by $\bar{X}_{i}$ the projected vector fields. A new version of theorem 2.2 in $(a, b)$ coordinates holds: 
Theorem 2.3. $\quad$ i) $\pi_{i}$ are Poisson tensors, $i \geq 0$;

ii) the functions $\bar{H}_{i}, i \geq 0$, are in involution with respect to all $\pi_{j}$;

iii) $\bar{X}_{i}\left(\bar{H}_{j}\right)=(1+i+j) \bar{H}_{i+j}, \quad i, j \geq 0$;

iv) $\mathcal{L}_{\bar{X}_{i}} \pi_{j}=(j-i-1) \pi_{i+j}, \quad i, j \geq 0$;

v) $\left[\bar{X}_{i}, \bar{X}_{j}\right]=(j-i) \bar{X}_{i+j}, \quad i, j \geq 0$;

vi) $\pi_{j} \nabla \bar{H}_{i}=\pi_{j-1} \nabla \bar{H}_{i+1}, \quad i \geq 0, j \geq 1$.

We call the hierarchies of Poisson tensors, master symmetries and Hamiltonians given by theorems 2.2 and 2.3 , in $(p, q)$ and $(a, b)$ coordinates respectively, the positive relativistic Toda hierarchies.

\section{The Negative RTL Hierarchy}

Our aim is to show that the relations of theorems 2.2 and 2.3 hold for any integer value of the index. For this purpose we define the negative relativistic Toda hierarchy. When the tensors are projected into the $(a, b)$ space we obtain rational Poisson brackets. We imitate the techniques of [3].

Let $\mathcal{N}$ be the inverse of the positive recursion operator $\mathcal{R}$,

$$
\mathcal{N}=\mathcal{R}^{-1}=\Lambda_{0}\left(\Lambda_{1}\right)^{-1}
$$

which is given in matrix form by $\mathcal{N}=\left(\begin{array}{cc}A & B \\ C & A^{T}\end{array}\right)$, where $A^{T}$ stands for the transpose of $A$ and the matrices $A=\left(a_{i j}\right), B=\left(b_{i j}\right)$ and $C=\left(c_{i j}\right)$, with $1 \leq i, j \leq N$, are defined by

$$
\begin{gathered}
\left\{\begin{aligned}
a_{i j} & =0, \quad j<i \\
a_{i i} & =e^{-p_{i}}+e^{q^{i-1}-q^{i}-p_{i}} \\
a_{i+1, i}= & e^{q^{i-1}-q^{i}-p_{i}} \\
a_{i+2, i}= & a_{i+3, i}=\cdots=a_{N, i}=e^{q^{i-1}-q^{i}-p_{i}}-e^{q-q^{i+1}-p_{i+1},},
\end{aligned}\right. \\
\left\{\begin{array}{r}
b_{i j}=-b_{j i} \\
b_{i+1, i}=b_{i+2, i}=\cdots=b_{N, i}=e^{-p_{i}}+e^{q^{i-1}-q^{i}-p_{i}}, \\
c_{i, i+1}=c_{i+1, i}=e^{q^{i}-q^{i+1}-p_{i+1}}
\end{array}\right.
\end{gathered}
$$

and all the others entries $c_{i j}$ are zero.

Let us denote by $K_{0}$ the conformal symmetry $X_{0}$,

$$
K_{0}=\sum_{i=1}^{N} \frac{\partial}{\partial p_{i}}=X_{0},
$$


and by $K_{i}$ the vector fields $K_{i}=\mathcal{N}^{i} K_{0}, i=0,1,2, \ldots$ These vector fields are master symmetries.

We next apply Oevel's theorem to the RTL, using the negative recursion operator $\mathcal{N}$ and the conformal symmetry $K_{0}$. Since

$$
\mathcal{L}_{K_{0}} \Lambda_{1}=0, \quad \mathcal{L}_{K_{0}} \Lambda_{0}=-\Lambda_{0}, \quad K_{0}\left(H_{1}\right)=2 H_{1},
$$

the constants are, in this case,

$$
\alpha_{\mathcal{N}}=0, \quad \beta_{\mathcal{N}}=-1, \quad \gamma_{\mathcal{N}}=2
$$

We remark that, obviously, $\alpha_{\mathcal{N}}=\beta$ and $\beta_{\mathcal{N}}=\alpha$.

Using the convention $X_{-i}=K_{i}$, for $i \geq 0$, we compute

$$
\left[X_{-i}, X_{-j}\right]=\left[K_{i}, K_{j}\right]=\left(\beta_{\mathcal{N}}-\alpha_{\mathcal{N}}\right)(j-i) K_{i+j}=(i-j) X_{-i-j},
$$

and letting $m=-i$ and $n=-j$, we obtain

$$
\left[X_{m}, X_{n}\right]=(n-m) X_{m+n}, \quad m, n \in \mathbb{Z}^{-} .
$$

The same relation holds in $(a, b)$-coordinates:

$$
\left[\bar{X}_{m}, \bar{X}_{n}\right]=(n-m) \bar{X}_{m+n}, \quad m, n \in \mathbb{Z}^{-} .
$$

Consider the following equalities (see [10]) which involve the recursion operator $\mathcal{R}$ and its inverse $\mathcal{N}$,

$$
\begin{gathered}
\mathcal{L}_{X_{0}} \mathcal{R}=\mathcal{L}_{X_{0}}\left(\Lambda_{1} \Lambda_{0}^{-1}\right)=(\beta-\alpha) \mathcal{R} \\
\mathcal{L}_{X_{0}} \mathcal{N}=\mathcal{L}_{X_{0}} \mathcal{R}^{-1}=(\alpha-\beta) \mathcal{R}^{-1}=(\alpha-\beta) \mathcal{N} .
\end{gathered}
$$

Using these relations we obtain, for $j \in \mathbb{N}$,

$$
\mathcal{L}_{X_{0}} \mathcal{R}^{j}=j(\beta-\alpha) \mathcal{R}^{j} \quad \text { and } \quad \mathcal{L}_{X_{0}} \mathcal{N}^{j}=j(\alpha-\beta) \mathcal{N}^{j} .
$$

Also, taking into account that $\mathcal{R}$ and $\mathcal{N}$ are torsionless tensors, we have, for any vector field $X$,

$$
\mathcal{L}_{N^{i} X} \mathcal{R}^{j}=N^{i}\left(\mathcal{L}_{X} \mathcal{R}^{j}\right), i, j \in \mathbb{N}
$$


Relations (22)-(25) allow us to compute the bracket of two master symmetries $K_{i}=X_{-i}$ and $X_{j}$, one in the negative hierarchy and the second in the positive one [3]. The result is

$$
\left[X_{-i}, X_{j}\right]=\left[\mathcal{N}^{i} X_{0}, \mathcal{R}^{j} X_{0}\right]=(j+i)(\beta-\alpha) X_{j-i} .
$$

In the case of the RTL, with $\alpha=1$ and $\beta=0,(26)$ turns to

$$
\left[X_{-i}, X_{j}\right]=(j+i) X_{j-i} .
$$

This relation also holds in $(a, b)$-coordinates and we conclude that (20) and (21) hold for any integer value of the index.

We can establish the following theorem.

Theorem 3.1. The conclusions of Theorems 2.2 and 2.3 hold for any integer value of the index.

Proof: The conditions $i$ ) and $i$ i) of theorem 2.2 are a direct consequence of Oevel's theorem and the properties of the recursion operators $\mathcal{R}$ and $\mathcal{N}$ [8]. The map $F$ being a Poisson morphism, $i$ ) of theorem 2.3 follows from $i$ ) of theorem 2.2. The proof of $i$ ) of theorem 2.3 is the same as the corresponding in the case of Toda system [3].

Regarding condition $\mathrm{iii}$ ) of theorem 2.2 , the only case we have to verify is $X_{-j}\left(H_{i}\right)$, with $i, j>0$. Consider $i \geq j$ (the other case is similar),

$$
\begin{aligned}
X_{-j}\left(H_{i}\right) & =\left\langle\mathcal{R}^{i}\left(d H_{0}\right), \mathcal{N}^{j} X_{0}\right\rangle=\left\langle d H_{0}, \mathcal{R}^{i-j}\right\rangle \\
& =X_{i-j}\left(H_{0}\right)=(1+i-j) H_{i-j} .
\end{aligned}
$$

To prove condition $\mathrm{iii}$ ) of theorem 2.3, involving the master symmetries $\bar{X}_{i}$, the arguments used in [3] in the case of the (classical) Toda lattice, can be applied for the RTL to prove that if $\lambda$ is an eigenvalue of the matrix $L$, the equation $\bar{X}_{n}(\lambda)=\lambda^{n+1}$ holds for $n \in \mathbb{Z}$. With $\bar{H}_{j}=\frac{1}{j+1} \operatorname{tr} L^{j+1}$, we obtain, for $n<0, j \geq 0$ and $j \neq-1-n$,

$$
\begin{aligned}
\bar{X}_{n}\left(\bar{H}_{j}\right) & =\frac{1}{j+1} \sum_{k=1}^{N} \bar{X}_{n}\left(\lambda_{k}^{j+1}\right) \\
& =(1+n+j) \bar{H}_{n+j}
\end{aligned}
$$


we conclude that

$$
\bar{X}_{n}\left(\bar{H}_{m}\right)=(1+m+n) \bar{H}_{n+m}
$$

holds for any $n, m \in \mathbb{Z}$.

Let us now show that conditions $i v$ ) of theorems 2.2 and 2.3 hold for negative indices. We set $W_{j}=\Lambda_{1-j}$ and for $i, j \geq 0$, we compute, using Oevel's theorem,

$$
\mathcal{L}_{X_{-i}} \Lambda_{-j}=\mathcal{L}_{K_{i}} W_{1+j}=\left(\beta_{\mathcal{N}}+\left(\beta_{\mathcal{N}}-\alpha_{\mathcal{N}}\right)(j-i)\right) W_{1+j+i}
$$

which gives,

$$
\mathcal{L}_{X_{-i}} \Lambda_{-j}=(-1+i-j) \Lambda_{-i-j} .
$$

Letting $m=-i$ and $n=-j$, we obtain

$$
\mathcal{L}_{X_{m}} \Lambda_{n}=(n-m-1) \Lambda_{m+n}, \quad m, n \in \mathbb{Z}^{-} .
$$

Finally, using the same technique as before, we can compute $\mathcal{L}_{X_{-i}} \Lambda_{j}, i, j \geq$ 0 , and we conclude that (29) holds for all $m, n \in \mathbb{Z}$.

Switching to $(a, b)$-coordinates, we obtain

$$
\mathcal{L}_{\bar{X}_{m}} \pi_{n}=(n-m-1) \pi_{m+n}, \quad m, n \in \mathbb{Z} .
$$

The condition $v$ ) of both theorems was already proved. Finally, the proofs of vi) of theorems 2.2 and 2.3 are similar to those in the case of the Toda system, see [3].

Remark 3.2. As it was remarked in [3], $\bar{H}_{n}=\frac{1}{n+1} \operatorname{tr} L^{n+1}$ is undefined for $n=-1$. We have $\bar{X}_{-1}\left(\bar{H}_{0}\right)=N$ and, more generally, $\bar{X}_{-n}\left(\bar{H}_{n-1}\right)=N$. If we define

$$
\bar{X}_{m}\left(\bar{H}_{-1}\right)=\lim _{n \rightarrow-1}\left(\bar{X}_{m}\left(\bar{H}_{n}\right)\right),
$$

then 


$$
\begin{aligned}
\bar{X}_{m}\left(\bar{H}_{-1}\right) & =\lim _{n \rightarrow-1}\left((1+m+n) \bar{H}_{n+m}\right) \\
& =m \bar{H}_{m-1} .
\end{aligned}
$$

Let us now show that the master symmetries $X_{n}, n \in \mathbb{Z}$, can be obtained once the hierarchy of Poisson tensors is known. In other words, we will show that all $X_{n}$ are Hamiltonian vector fields associated with the same function $f$, w.r.t. the Poisson tensors of the hierarchy.

Consider the function $f=\sum_{i=1}^{N} q^{i}$. Then $X_{n}(f)=0$, for all $n \in \mathbb{Z}$. In fact,

$$
X_{0}(f)=\sum_{i=1}^{N} \frac{\partial f}{\partial p_{i}}=0
$$

and since $X_{1}=\mathcal{R} X_{0}$ is given by $[9]$

$$
\begin{aligned}
X_{1} & =\sum_{i=1}^{N}\left((1-i)\left(e^{q^{i}-q^{i+1}+p_{i}}+e^{p_{i}}\right)+\sum_{j=i+1}^{N}\left(e^{q^{j}-q^{j+1}+p_{j}}+e^{p_{j}}\right)\right) \frac{\partial}{\partial q^{i}} \\
& +\sum_{i=1}^{N}\left(e^{p_{i}}+i e^{q^{i}-q^{i+1}+p_{i}}+(2-i) e^{q^{i-1}-q^{i}+p_{i-1}}\right) \frac{\partial}{\partial p_{i}},
\end{aligned}
$$

where, by convention, $q^{0}=-\infty$ and $q^{n+1}=+\infty$, we compute

$$
X_{1}(f)=\sum_{i=1}^{n}\left((1-i)\left(e^{q^{i}-q^{i+1}+p_{i}}+e^{p_{i}}\right)+\sum_{j=i+1}^{n}\left(e^{q^{j}-q^{j+1}+p_{j}}+e^{p_{j}}\right)\right)=0 .
$$

Similarly, one proves that $X_{2}(f)=0$. By induction we prove that $X_{n}(f)=0$, for all $n \in \mathbb{N}$ : if $X_{n-1}(f)=0$, then

$$
\begin{aligned}
X_{n}(f) & =\frac{1}{n-2}\left[X_{1}, X_{n-1}\right](f) \\
& =\frac{1}{n-2}\left(X_{1}\left(X_{n-1}(f)\right)-X_{n-1}\left(X_{1}(f)\right)\right) \\
& =0 .
\end{aligned}
$$


For the negative hierarchy of master symmetries, the proof is similar, with $X_{-n}=K_{n}$. The vector field $X_{-1}=K_{1}$ is given by

$$
\begin{aligned}
X_{-1} & =\sum_{i=1}^{N}\left(\sum_{j=1}^{i-1}\left(e^{-p_{j}}+e^{q^{j-1}-q^{j}-p_{j}}\right)+(i-N)\left(e^{-p_{i}}+e^{q^{i-1}-q^{i}-p_{i}}\right)\right) \frac{\partial}{\partial q^{i}} \\
& +\sum_{i=1}^{N}\left(e^{-p_{i}}+(N+1-i) e^{q^{i-1}-q^{i}-p_{i}}+(1+i-N) e^{q^{i}-q^{i+1}-p_{i+1}}\right) \frac{\partial}{\partial p_{i}}
\end{aligned}
$$

and we compute

$$
\begin{aligned}
X_{-1}(f) & =\sum_{i=1}^{N}\left(\sum_{j=1}^{i-1}\left(e^{-p_{j}}+e^{q^{j-1}-q^{j}-p_{j}}\right)+(i-N)\left(e^{-p_{i}}+e^{q^{i-1}-q^{i}-p_{i}}\right)\right) \\
& =0 .
\end{aligned}
$$

Now, we remark that $X_{0}=\sum_{i=1}^{N} \frac{\partial}{\partial p_{i}}$ is the Hamiltonian vector field of $f$ with respect to $\Lambda_{1}$, i.e. $X_{0}=-\left[\Lambda_{1}, f\right]$, where [,] stands for the Schouten bracket [7]. Next, we show that every master symmetry $X_{n}$ is an Hamiltonian vector field of $f$.

Proposition 3.3. For any $n \in \mathbb{Z}, X_{n}=-\left[\Lambda_{n+1}, f\right]$.

Proof: We proceed by induction, starting with the positive hierarchy ( $n \in$ $\mathbb{N}_{0}$ ). For $n=0$, we compute

$$
-\left[\Lambda_{1}, f\right]=-\sum_{i, j=1}^{N}\left[\frac{\partial}{\partial q^{i}} \wedge \frac{\partial}{\partial p_{i}}, q^{j}\right]=X_{0} .
$$

If $X_{n-1}=-\left[\Lambda_{n}, f\right]$, then

$$
\begin{aligned}
X_{n} & =\frac{1}{n-2}\left[X_{1}, X_{n-1}\right] \\
& =-\frac{1}{n-2}\left[X_{1},\left[\Lambda_{n}, f\right]\right] .
\end{aligned}
$$

Using the generalized Jacobi identity for the Schouten bracket [7], we obtain 


$$
\begin{aligned}
X_{n} & =-\frac{1}{n-2}\left[f,\left[X_{1}, \Lambda_{n}\right]\right] \\
& =-\frac{1}{n-2}\left[\mathcal{L}_{X_{1}} \Lambda_{n}, f\right] \\
& =-\left[\Lambda_{n+1}, f\right] .
\end{aligned}
$$

If $n<0$, the proof is similar.

Remark 3.4. In a similar way, it is easy to prove that the Hamiltonians $H_{i}$ are also determined from the knowledge of the Poisson brackets associated with $\left(\Lambda_{i}\right), n \in \mathbb{Z}$ and the function $f$.

Next we exhibit, in $(a, b)$-coordinates, some rational Poisson brackets and master symmetries of the negative relativistic Toda hierarchy. We present the case $N=3$, in order to simplify the notation. The bracket corresponding to the Poisson tensor $\pi_{-1}$ on $\mathbb{R}^{2 N-1}$, which is the projection of $\Lambda_{-1}$ (by $F$ ), is the following:

$$
\begin{aligned}
\left\{a_{1}, a_{2}\right\}_{-1} & =\frac{1}{b_{1} b_{2} b_{3}} a_{1} a_{2}\left(b_{1}-b_{2}+b_{3}\right) \\
\left\{a_{1}, b_{1}\right\}_{-1} & =\frac{1}{b_{1} b_{2} b_{3}} a_{1}\left(b_{2} b_{3}+a_{1} b_{3}\right) \\
\left\{a_{1}, b_{2}\right\}_{-1} & =\frac{1}{b_{1} b_{2} b_{3}}\left(a_{1} a_{2}\left(b_{2}-b_{1}\right)-a_{1} b_{3}\left(a_{1}+b_{1}\right)\right) \\
\left\{a_{1}, b_{3}\right\}_{-1} & =\frac{1}{b_{1} b_{2} b_{3}}\left(-a_{1} a_{2} b_{3}\right) \\
\left\{a_{2}, b_{1}\right\}_{-1} & =\frac{1}{b_{1} b_{2} b_{3}} a_{1} a_{2} b_{1} \\
\left\{a_{2}, b_{2}\right\}_{-1} & =\frac{1}{b_{1} b_{2} b_{3}}\left(a_{2} b_{1}\left(a_{2}+b_{3}\right)+a_{1} a_{2}\left(b_{3}-b_{2}\right)\right) \\
\left\{a_{2}, b_{3}\right\}_{-1} & =\frac{1}{b_{1} b_{2} b_{3}} a_{2} b_{1}\left(-a_{2}-b_{2}\right) \\
\left\{b_{1}, b_{2}\right\}_{-1} & =\frac{1}{b_{1} b_{2} b_{3}}\left(a_{1} b_{1}\left(a_{2}+b_{3}\right)+a_{1} b_{3}\left(a_{1}+b_{2}\right)\right) \\
\left\{b_{2}, b_{3}\right\}_{-1} & =\frac{1}{b_{1} b_{2} b_{3}}\left(a_{2} b_{1}\left(a_{2}+b_{3}\right)+a_{1} a_{2} b_{3}+a_{2} b_{1} b_{2}\right) .
\end{aligned}
$$


The denominator $b_{1} b_{2} b_{3}$ is equal to $\operatorname{det} L$, where $L$ is the matrix (6) of the Lax pair $(L, M)$. So the Poisson tensor $\pi_{-1}$ is defined on the open dense set $\operatorname{det} L \neq 0$.

The vector field $X_{-1}$ in $(p, q)$-coordinates is given by (31). The expression of $X_{-2}$ in the same coordinates is quite complicated. The projections of $X_{-1}$ and $X_{-2}$ in $(a, b)$ coordinates, for $N=3$, are the following:

$X_{-1}=\left(-\frac{2 a_{1}}{b_{1}}+\frac{a_{1}}{b_{2}}\right) \frac{\partial}{\partial a_{1}}-\frac{a_{2}}{b_{2}} \frac{\partial}{\partial a_{2}}+\left(1-\frac{a_{1}}{b_{2}}\right) \frac{\partial}{\partial b_{1}}+\left(1+\frac{2 a_{1}}{b_{1}}\right) \frac{\partial}{\partial b_{2}}+\left(1+\frac{a_{2}}{b_{2}}\right) \frac{\partial}{\partial b_{3}}$

and

$$
X_{-2}=\sum_{i=1}^{2} u_{i} \frac{\partial}{\partial a_{i}}+\sum_{i=1}^{3} v_{i} \frac{\partial}{\partial b_{i}}
$$

with

$$
\begin{aligned}
& u_{1}=-\frac{2 a_{1}}{b_{1}^{2}}+\frac{a_{1}}{b_{2}^{2}}-\frac{2 a_{1}}{b_{1} b_{2}}-\frac{2 a_{1}^{2}}{b_{1}^{2} b_{2}}+\frac{a_{1}^{2}}{b_{1} b_{2}^{2}}+\left(\frac{a_{1}}{b_{3}}+\frac{a_{1} a_{2}}{b_{2} b_{3}}\right)\left(\frac{1}{b_{2}}-\frac{1}{b_{1}}\right) \\
& u_{2}=-\frac{a_{2}}{b_{2}^{2}}+\frac{a_{2}}{b_{1} b_{2}}\left(1-\frac{a_{1}}{b_{2}}\right)-\frac{a_{2}}{b_{2} b_{3}}\left(2+\frac{a_{2}}{b_{2}}\right)-\frac{a_{2}}{b_{1} b_{3}}\left(1+\frac{a_{1}}{b_{2}}\right) \\
& v_{1}=\frac{1}{b_{1}}-\frac{a_{1}}{b_{2}^{2}}-\frac{a_{1}^{2}}{b_{1} b_{2}^{2}}-\frac{a_{1}}{b_{2} b_{3}}-\frac{a_{1} a_{2}}{b_{2}^{2} b_{3}} \\
& v_{2}=\frac{2 a_{1} a_{2}}{b_{1} b_{2} b_{3}}+\frac{a_{1}+a_{2}}{b_{1} b_{3}}+\frac{a_{1}}{b_{1} b_{2}}+\frac{a_{2}}{b_{2} b_{3}}+\frac{a_{1}^{2}}{b_{1}^{2} b_{2}}+\frac{2 a_{1}}{b_{1}^{2}}+\frac{1}{b_{2}}\left(1+\frac{a_{1}}{b_{1}}\right)^{2} \\
& v_{3}=-\frac{a_{2}}{b_{1} b_{2}}+\frac{a_{1} a_{2}}{b_{1} b_{2}^{2}}+\frac{a_{2}}{b_{2}^{2}}+\frac{1}{b_{3}}\left(1+\frac{a_{2}}{b_{2}}\right)^{2} .
\end{aligned}
$$

The function $\operatorname{det} L=b_{1} b_{2} b_{3}$ is a Casimir of $\pi_{1}$. On the other hand, $\operatorname{tr} L=$ $H_{0}$ is a Casimir of $\pi_{0}$. More generally, we can prove:

Proposition 3.5. The function $\operatorname{tr} L^{1-n}$ is a Casimir of $\pi_{n}$, for all $n \in \mathbb{Z} \backslash\{1\}$.

Proof: For $n=2$, the technique of [2] (p. 5525) can be applied word for word to show that $\operatorname{tr} L^{-1}$ is a Casimir of $\pi_{2}$, i.e. $\pi_{2} \nabla\left(\operatorname{tr} L^{-1}\right)=0$. But $\operatorname{tr} L^{-1}=-H_{-2}$. So we get $\pi_{2} \nabla H_{-2}=0$.

By theorem 2.3, 


$$
0=\pi_{2} \nabla H_{-2}=\pi_{3} \nabla H_{-3}=\ldots
$$

and the result is proved for all $n>1$. For $n=0$, as we already remarked, $\operatorname{tr} L=H_{0}$ is a Casimir of $\pi_{0}$. So, $\pi_{0} \nabla H_{0}=0$ and using again the Lenard relations of theorem 2.3 , we obtain

$$
0=\pi_{0} \nabla H_{0}=\pi_{-1} \nabla H_{1}=\pi_{-2} \nabla H_{2} \ldots
$$

and the result is proved for $n<1$.

\section{References}

[1] M. Bruschi and O. Ragnisco, Recursion operator and Bäcklund transformations for the Ruijsenaars Toda lattice, Phys. Lett. A (129) (1988) 21-25.

[2] P. A. Damianou, Multiple Hamiltonian structures for Toda-type systems, J. Math. Phys. (35) (1994) 5511-5541.

[3] P.A. Damianou, The Negative Toda Hierarchy and Rational Poisson Brackets, J. Geom. Phys. (45) (2003) 184-202.

[4] P. A. Damianou, On the bi-Hamiltonian structure of Bogoyavlensky-Toda lattices, Technical Report TR/09/2002, Department of Mathematics and Statistics, University of Cyprus, (2002).

[5] A. Das, and S. Okubo, A systematic study of the Toda lattice, Ann. Physics. (190) (1989) $215-232$.

[6] R. L. Fernandes, On the mastersymmetries and bi-Hamiltonian structure of the Toda lattice, J. Phys. A (26) (1993) 3797-3803.

[7] J.-L. Koszul, Crochet de Schouten-Nijenhuis et cohomologie, in Élie Cartan et les mathématiques d'aujourd'hui, Astérisque, numéro hors série (1985)

[8] F. Magri and C. Morosi, A geometrical characterisation of integrable Hamiltonian systems through the theory of Poisson-Nijenhuis manifolds, Quaderno S 19, Univ. de Milano, (1984).

[9] J. M. Nunes da Costa and C.-M. Marle, Master symmetries and bi-Hamiltonian structures for the relativistic Toda lattice, J. Phys. A (30) (1997) 7551-7556.

[10] W. Oevel, A geometrical approach to integrable systems admitting time dependent invariants, in Topics in Soliton Theory and Exactly Solvable Nonlinear Equations, ed. M. Ablowitz, B. Fuchssteiner and M. Kruskal, World Scientific, Singapore, (1987).

[11] W. Oevel, B. Fuchssteiner, H. Zhang and O. Ragnisco, Mastersymmetries, angle variables, and recursion operator of the relativistic Toda lattice, J. Math. Phys. (30) (1989) 2664-2670.

[12] Yu. B. Suris, On the bi-Hamiltonian structure of Toda and relativistic Toda lattices, Phys. Lett. A (180) (1993) 419-429.

[13] S. N. Ruijsenaars, Relativistic Toda Systems, Commun. Math. Phys. (133) (1990) 217-247.

\section{J. M. Nunes DA Costa}

Departamento de Matemática, Universidade de Coimbra, Apartado 3008, 3001-454 CoimBra, Portugal, Email: Jmcosta@mat.uc.Pt

\section{P. A. Damianou}

Department of Mathematics and Statistics, University of Cyprus, P. O. Box 20537,1678

Nicosia, Cyprus, Email: DAMiANOU@UCy.AC.CY 